\title{
THE RESEARCH JOURNAL TRANSPORT: PEER-REVIEWING PROCESS IN 2013
}

\author{
Olegas Prentkovskis ${ }^{1}$, Raimundas Junevičius ${ }^{2}$ \\ ${ }^{1}$ Editor-in-Chief, E-mail: olegas.prentkovskis@vgtu.lt \\ ${ }^{2}$ Managing (Associate) Editor, E-mail: raimundas.junevicius@vgtu.lt \\ The research journal TRANSPORT is published by Vilnius Gediminas Technical University \\ in partnership with the Lithuanian Academy of Science and Taylor \& Francis
}

3 December 2013

As one of the greatest values of humanity, freedom of movement depends on a well-developed system of transport. Moreover, reliable and properly organized transport services are crucial for industry, construction and agriculture, which positions the transport sector among the most important national economic drivers.

Aiming to ensure harmonious functioning of separate elements as well as the entire system of transport, scientists from various countries undertake research efforts that result in significant developments.

The Editorial Board of the research journal TRANSPORT is proud to offer research articles on a wide variety of such important topics as:

- transport policy;

- fundamentals of the transport system;

- technologies for passenger and freight transportation using road, railway, inland waterway, sea and air transport;

- technologies for multimodal transportation and logistics;

- loading technologies;

- roads, railways, airports, ports and pipeline transport;

- industrial and technological transport;

- agricultural motor vehicles;

- traffic safety;

- transport flows;

- environmental protection;

- design, manufacture and exploitation of motor vehicles;

- transport energetics;

- fuels, lubricants and maintenance materials;

- customs and transport;

- insurance;

- transport information technologies;

- transport economics and management;

- transport standards;

- transport education;

- transport history, etc.

The research journal TRANSPORT is a peer-reviewed journal. Since 2010, to peer-review manuscript submissions, the research journal TRANSPORT has been using the interactive system ScholarOne Manuscripts (formerly known as Manuscript Central; http://mc.manuscriptcentral.com/stra).

All manuscripts submitted through ScholarOne Manuscripts to the research journal TRANSPORT are peer-reviewed by members of the Editorial Board or appointed experts. 
The minimum number of score sheets (peer-review forms filled by reviewers for each submitted manuscript in 2013) amounts to three and the average number of score sheets (peer-review forms) is 3.8.

Decision ratio of submitted manuscripts in 2013:

\begin{tabular}{l|c|c|c|c|c}
\hline Decision & Accept & Minor revision & Major revision & Reject & Reject-inappropriate \\
\hline Original submission & - & $22.73 \%$ & $28.41 \%$, & $14.77 \%$ & $34.09 \%$ \\
\hline Revised submission (resubmission) & $56.52 \%$ & $23.19 \%$ & $15.94 \%$ & $4.35 \%$ & - \\
\hline All submissions & $24.84 \%$ & $22.93 \%$ & $22.93 \%$ & $10.19 \%$ & $19.11 \%$ \\
\hline
\end{tabular}

Success and quality of the research journal TRANSPORT depends to a large extent on the activity and performance of its Editorial Board and Reviewers. Reviewers - scientists who volunteer to contribute to improvement of the quality of transport engineering and transport management fields by guiding authors in their efforts to publish their research outputs - help enlarging the scientific content of the research journal TRANSPORT, for which we feel truly grateful.

Thus, on behalf of the Editorial Board of the research journal TRANSPORT we would like to express our gratitude to the following reviewers for their important contribution to the quality of the journal in 2013 (papers submitted in 2011-2013 and published in 2013):

\begin{tabular}{|c|c|c|c|}
\hline Name & Surname & Organisation & Country \\
\hline Demi & Chung & \multirow[t]{5}{*}{ The University of Sydney } & \multirow[t]{5}{*}{ Australia } \\
\hline Geoffrey & Clifton & & \\
\hline Rico & Merkert & & \\
\hline Corinne & Mulley & & \\
\hline Peter & Stopher & & \\
\hline Cathy & Macharis & Free University of Brussels & \multirow[t]{4}{*}{ Belgium } \\
\hline Hongying & Fei & \multirow[t]{3}{*}{ University of Mons } & \\
\hline Georges & Kouroussis & & \\
\hline Olivier & Verlinden & & \\
\hline Mário Ricardo & Gongora-Rubio & IPT - Institute for Technological Research & \multirow[t]{2}{*}{ Brazil } \\
\hline Celso & Morooka & State University of Campinas & \\
\hline Maureen & Reed & Ryerson University & Canada \\
\hline Liang & Gao & Beijing Jiaotong University & \multirow[t]{3}{*}{ China } \\
\hline Yong-Gang & Wang & Chang'an University & \\
\hline Ying-En & $\mathrm{Ge}$ & Dalian University of Technology & \\
\hline Mirano & Hess & \multirow[t]{2}{*}{ University of Rijeka } & \multirow[t]{2}{*}{ Croatia } \\
\hline Svjetlana & Hess & & \\
\hline Jiř́í & Chlebek & Brno University of Technology & \multirow{11}{*}{$\begin{array}{l}\text { Czech } \\
\text { Republic }\end{array}$} \\
\hline Ladislav & Bína & \multirow[t]{6}{*}{ Czech Technical University in Prague } & \\
\hline Vít & Janoš & & \\
\hline Libor & Beneš & & \\
\hline Josef & Kocourek & & \\
\hline Dagmar & Kočárková & & \\
\hline Kristýna & Neubergová & & \\
\hline Jan & Černý & University of Economics in Prague & \\
\hline Michael & Lata & \multirow[t]{2}{*}{ University of Pardubice } & \\
\hline Jaroslav & Matuška & & \\
\hline Jakub & Gottvald & VÍTKOVICE ÚAM, Inc. & \\
\hline Andrus & Aavik & \multirow[t]{2}{*}{ Tallinn University of Technology } & \multirow[t]{2}{*}{ Estonia } \\
\hline Dago & Antov & & \\
\hline
\end{tabular}




\begin{tabular}{|c|c|c|c|}
\hline Name & Surname & Organisation & Country \\
\hline Eric & Bruun & \multirow[t]{3}{*}{ Aalto University } & \multirow[t]{7}{*}{ Finland } \\
\hline Jerzy & Matusiak & & \\
\hline Jakub & Montewka & & \\
\hline Pentti & Kujala & Helsinki University of Technology & \\
\hline Ulla & Tapaninen & \multirow[t]{2}{*}{ University of Turku } & \\
\hline Sari & Repka & & \\
\hline Wladimir & Segercrantz & - & \\
\hline Michele & Acciaro & Kühne Logistics University & Germany \\
\hline George & Giannopolos & \multirow{2}{*}{ Aristotle University of Thessaloniki } & \multirow[t]{5}{*}{ Greece } \\
\hline Zisis & Samaras & & \\
\hline Josep Maria & Salanova Grau & Hellenic Institute of Transport & \\
\hline Nikitas & Nikitakos & \multirow{2}{*}{ University of the Aegean } & \\
\hline Athanasios & Pallis & & \\
\hline Ákos & Bereczky & \multirow[t]{6}{*}{ Budapest University of Technology and Economics } & \multirow[t]{7}{*}{ Hungary } \\
\hline Zoltan & Bokor & & \\
\hline Tamás & Markovits & & \\
\hline Tamás & Péter & & \\
\hline Katalin & Tanczos & & \\
\hline Ádám & Török & & \\
\hline Máté & Zöldy & MOL DS Product Development & \\
\hline Cristina & Pronello & Polytechnic University of Turin & \multirow[t]{4}{*}{ Italy } \\
\hline Gabriella & Mazzulla & University of Calabria & \\
\hline Mario & De Luca & \multirow[t]{2}{*}{ University of Napoli Federico II } & \\
\hline Gianluca & Dell'Acqua & & \\
\hline Mareks & Mezītis & \multirow[t]{3}{*}{ Riga Technical University } & \multirow[t]{5}{*}{ Latvia } \\
\hline Jānis & Vìba & & \\
\hline Juris & Smirnovs & & \\
\hline Igor & Kabashkin & \multirow[t]{2}{*}{ Transport and Telecommunication Institute } & \\
\hline Irina & Yatskiv (Jackiva) & & \\
\hline Algirdas & Janulevičius & \multirow[t]{5}{*}{ Aleksandras Stulginskis University } & \multirow[t]{23}{*}{ Lithuania } \\
\hline Gvidonas & Labeckas & & \\
\hline Violeta & Makarevičienè & & \\
\hline Gediminas & Pupinis & & \\
\hline Stasys & Slavinskas & & \\
\hline Rolandas & Vitkūnas & International School of Law and Business & \\
\hline Žilvinas & Bazaras & \multirow[t]{13}{*}{ Kaunas University of Technology } & \\
\hline Jonas & Daunoras & & \\
\hline Vytautas & Gargasas & & \\
\hline Paulius & Griškevičius & & \\
\hline Jurga & Ilgakojyté-Bazarienè & & \\
\hline Janina & Jablonskytė & & \\
\hline Artūras & Keršys & & \\
\hline Robertas & Keršys & & \\
\hline Dainius & Martuzevičius & & \\
\hline Kęstutis & Pilkauskas & & \\
\hline Henrikas & Pranevičius & & \\
\hline Laurencas & Raslavičius & & \\
\hline Jonas & Sapragonas & & \\
\hline Arūnas & Andziulis & \multirow[t]{4}{*}{ Klaipèda University } & \\
\hline Mindaugas & Česnauskis & & \\
\hline Jolanta & Janutènienè & & \\
\hline Sergejus & Lebedevas & & \\
\hline
\end{tabular}




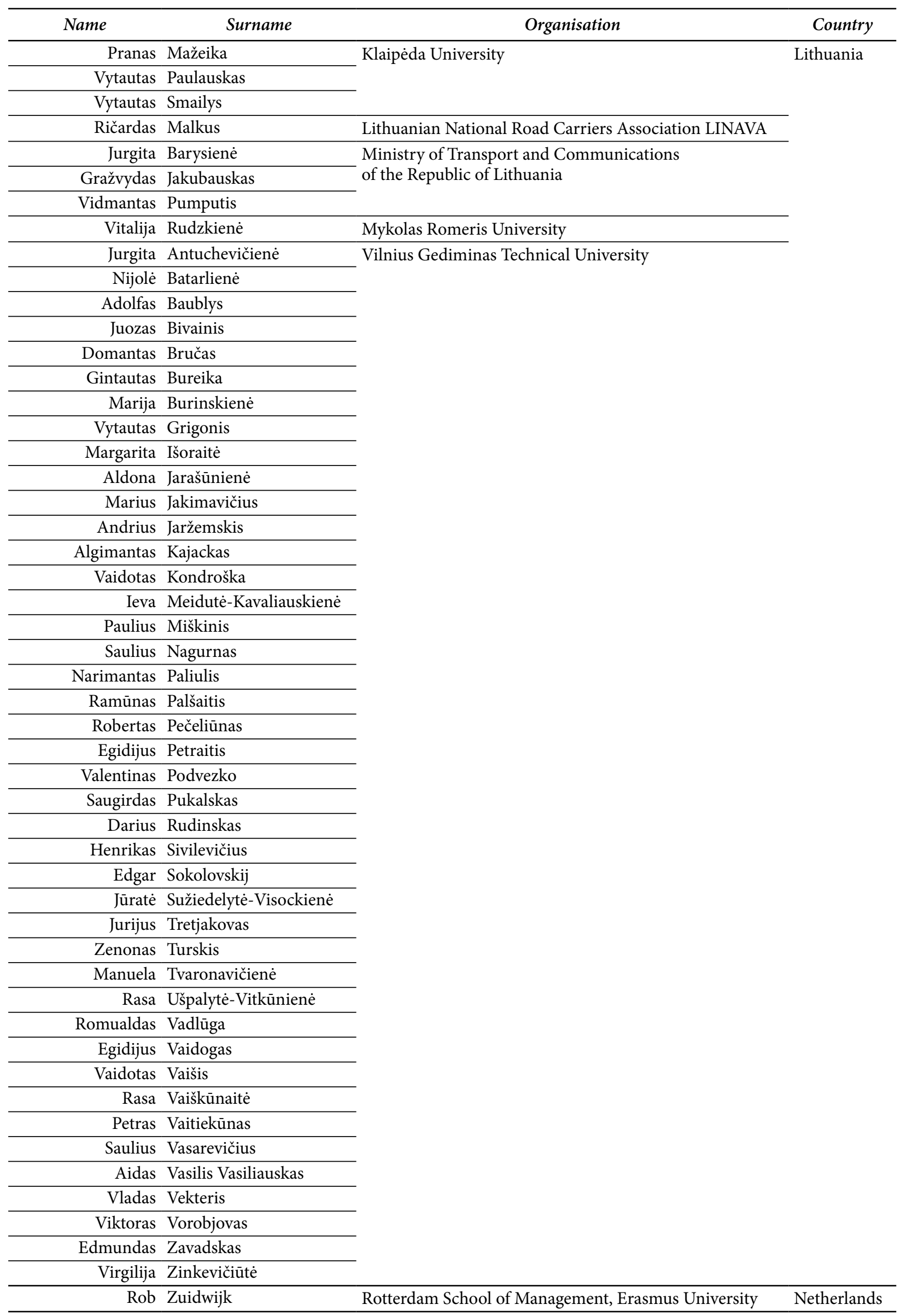




\begin{tabular}{|c|c|c|c|}
\hline Name & Surname & Organisation & Country \\
\hline Wojciech & Potkański & Institute of Aviation & Poland \\
\hline Gabriel & Nowacki & Military University of Technology & \\
\hline Jerzy & Paslawski & Poznan University of Technology & \\
\hline Marek & Sitarz & Silesian University of Technology & \\
\hline Leszek & Golonka & Wroclaw University of Technology & \\
\hline Ivan & Dyakov & Ulyanovsk State Technical University & $\begin{array}{l}\text { Russian } \\
\text { Federation }\end{array}$ \\
\hline Obrad & Babić & University of Belgrade & Serbia \\
\hline Olja & Cokorilo & & \\
\hline Jadranka & Jović & & \\
\hline Maksym & Starykov & Portek Systems \& Equipment Pte Ltd & Singapore \\
\hline Norbert & Adamko & University of Žilina & Slovakia \\
\hline Karel & Havel & & \\
\hline Michal & Koháni & & \\
\hline Peter & Matis & & \\
\hline Milan & Batista & University of Ljubljana & Slovenia \\
\hline Bojan & Beškovnik & & \\
\hline Jana & Šelih & & \\
\hline Elen & Twrdy & & \\
\hline Marijan & Žura & & \\
\hline Uroš & Klanšek & University of Maribor & \\
\hline Joaquim & Casal & Polytechnic University of Catalonia & Spain \\
\hline Pedro & Cantos & University of Valencia & \\
\hline Åsa & Forsman & Swedish National Road and Transport Research Institute & Sweden \\
\hline Erik & Wilhelm & Paul Scherrer Institute & Switzerland \\
\hline Aurimas & Vilkelis & Swiss Federal Institutes of Technology Zurich & \\
\hline Serhan & Tanyel & Dokuz Eylül University & Turkey \\
\hline Ali & Akgüngör & Kirikkale University & \\
\hline Andrii & Bieliatynskyi & National Aviation University & Ukraine \\
\hline Andrii & Grekhov & & \\
\hline Viktoriia & Ivannikova & & \\
\hline Nina & Kuzhel & & \\
\hline Iryna & Klymenko & National University of Transport & \\
\hline Rawindaran & Nair & Cardiff University & United \\
\hline Kathryn & Stewart & Edinburgh Napier University & Kingdom \\
\hline Marcus & Enoch & Loughborough University & \\
\hline Teresa & Lombardo & & \\
\hline David & Pitfield & & \\
\hline Caitlin & Cottrill & University of Aberdeen & \\
\hline John & Nelson & & \\
\hline Felix & Schmid & University of Birmingham & \\
\hline John & Kinuthia & University of Glamorgan & \\
\hline Karl & Ropkins & University of Leeds & \\
\hline Panos & Konstantopoulos & University of Nottingham & \\
\hline Neale & Blair & University of Ulster & \\
\hline Jing & Dong & Iowa State University & United States \\
\hline Kasthurirangan & Gopalakrishnan & & \\
\hline Qingang & Xiong & & \\
\hline Jose & Serpa & Miami Dade College & \\
\hline John & Bullough & Rensselaer Polytechnic Institute & \\
\hline Litao & Liu & Texas A \& M Transportation Institute & \\
\hline Leon & James & University of Hawai'i System & \\
\hline Qian & Zhou & University of Virginia & \\
\hline
\end{tabular}

